\title{
NUMBER OF REAL ROOTS OF A RANDOM TRIGONOMETRIC POLYNOMIAL ${ }^{1}$
}

\author{
K. FARAHMAND \\ University of Ulster \\ Department of Mathematics \\ Jordanstown, Co Antrim \\ BT37 OQB United Kingdom
}

\begin{abstract}
We study the expected number of real roots of the random equation $g_{1} \cos \theta+g_{2} \cos 2 \theta+\ldots+g_{n} \cos n \theta=K$ where the coefficients $g_{j}$ 's are normally distributed, but not necessarily all identical. It is shown that although this expected number is independent of the means of $g_{j}, j=1,2, \ldots, n$, it will depend on their variances. The previous works in this direction considered the identical distribution for the coefficients.
\end{abstract}

Key words: Number of real roots, number of level crossings, random trigonometric polynomial, Kac-Rice formula.

AMS (MOS) subject classifications: Primary, 60G99; Secondary 42BXX.

\section{INTRODUCTION}

Let $g_{1}(\omega), g_{2}(\omega), \ldots, g_{n}(\omega)$ be a sequence of independent normally distributed random variables defined on a probability space $(\Omega, \mathcal{A}, P)$, and $N(\alpha, \beta) \equiv N_{n, K}(\alpha, \beta)$ be the number of real roots of the equation $T(\theta)=K$ in the interval $\alpha \leq \theta \leq \beta$ where

$$
T(\theta) \equiv T_{n}(\theta, \omega)=\sum_{j=1}^{n} g_{j}(\omega) \cos j \theta .
$$

Some years ago Dunnage [2] assumed identical distribution for $g_{j}(\omega),(j=1,2, \ldots, n)$ and for case of $K=E\left(g_{j}\right)=0$ showed that $E N(0,2 \pi)$, the mathematical expectation of $N(0,2 \pi)$, is asymptotic to $2 n / \sqrt{3}$. Later Farahmand [4] found the same asymptotic formula when he considered the case of $K$ and $E\left(g_{j}\right)=\mu \neq 0$ as any constants. However, the persistence of this asymptotic formula is not typical for the other types of random polynomials. For instance, for the algebraic polynomial $Q(x)=\sum_{j=1}^{n} g_{j} x^{i}$ Ibragimov and Maslova [6] for the case $K=0$, $E\left(g_{j}\right)=\mu \neq 0$ obtained half of the real roots of the case $E\left(g_{j}\right)=0$. Farahmand [3] also found

\footnotetext{
${ }^{1}$ Received: December, 1991. Revised: September, 1992.
} 
a reduction in the number of real roots when he considered the case of $K \neq 0$ rather than $K=0$. Very recently in two interesting papers involving several new methods, Wilkins $[10,11]$ dramatically reduces the error term for the case of $K=0$, considerably improving the previous result.

In this paper, for the random trigonometric polynomial (1.1), we will study the case when the means and variances of the coefficients $g_{j}$ are not necessarily all equal. It will be shown that $E N(0,2 \pi)$ will be independent of $E\left(g_{j}\right)$ and $K$, but dependent on the $\operatorname{var}\left(g_{j}\right)$. For $\mu_{1}, \mu_{2}, \sigma_{1}^{2}$ and $\sigma_{2}^{2}$ as any bounded absolute constants, we prove the following theorem:

Theorem: If the coefficients $g_{j}, j=1,2, \ldots, n$ of $T(\theta)$ are normally distributed with means and variances $\mu_{1}$ and $\sigma_{1}^{2}>0$ for $1 \leq j \leq n_{1}$ and $\mu_{2}$ and $\sigma_{2}^{2}>0$ for $n_{1}<j \leq n$, respectively, then for any sequence of constants $K_{n} \equiv K$, such that $K^{2} /\left(n_{1} \sigma_{1}^{2}+n \sigma_{2}^{2}\right)$ tends to zero as $n_{1}$ tends to infinity, the mathematical expectation of the number of real roots of the equation $T(\theta)=K$ satisfies

$$
E N(0,2 \pi) \sim 2\left[\frac{n_{1}^{3} \sigma_{1}^{2}+\left(n^{3}-n_{1}^{3}\right) \sigma_{2}^{2}}{3\left\{n_{1} \sigma_{1}^{2}+\left(n-n_{1}\right) \sigma_{2}^{2}\right\}}\right]^{\frac{1}{2}} .
$$

Comparing this result with the algebraic case with non-identically distributed coefficients studied in [5], shows another interesting difference between these two types of polynomials. That is, for the algebraic case, the behavior of the number of real roots is dictated by the means and is independent of variances while, in contrast, for the trigonometric case, this number depends on the variances and not on the means. In the proof of the theorem, the assumption that $\mu_{1}, \mu_{2}, \sigma_{1}^{2}, \sigma_{2}^{2}$ are independent of $n$ is not necessary, but the gain in stating a marginally more general conclusion subject to a very ungainly hypothesis seems insignificant.

\section{PROOF OF THE THEOREM}

We first look at the polynomial $T(\theta)-K$ as a non-stationary normal process with $\lambda_{1}$ and $A^{2}$, say, as its mean and variance and $\lambda_{2}$ and $B^{2}$, say, as the mean and variance of its derivative, respectively. Then we can use Cramér and Leadbetter's [1, page 285] result for the level crossings of this type of process to get,

$$
E N(\alpha, \beta)=\int_{\alpha}^{\beta}(B / A)\left(1-C^{2} / A^{2} B^{2}\right)^{\frac{1}{2}} \phi\left(\lambda_{1} / A\right)[2 \phi(\eta)+\eta\{2 \Phi(\eta)-1\}] d \theta
$$

where

$$
C=\operatorname{COV}\left[\{T(\theta)-K\}, T^{\prime}(\theta)\right], \eta=\left(C \lambda_{1}-A^{2} \lambda_{2}\right) / A \Delta,
$$


and

$$
\Delta^{2}=A^{2} B^{2}-C^{2}, \Phi(t)=(2 \pi)^{-\frac{1}{2}} \int_{-\infty}^{t} \exp \left(-y^{2} / 2\right) d y
$$

$\phi(t)=\Phi^{\prime}(t)=(2 \pi)^{-\frac{1}{2}} \exp \left(-t^{2} / 2\right)$.

Now since $\Phi(t)=\frac{1}{2}+(\pi)^{-\frac{1}{2}} \operatorname{er} f(t / \sqrt{2})$ where $\operatorname{erf}(x)=\int_{-\infty}^{x} \exp \left(-t^{2}\right) d t$ from $(2.1)$ we have the extension of the Kac-Rice [7] formula

$$
\begin{gathered}
E N(\alpha, \beta)=\int_{\alpha}^{\beta}\left[\left(\Delta / \pi A^{2}\right) \exp \left\{-\left(B^{2} \lambda_{1}^{2}-2 C \lambda_{1} \lambda_{2}+A^{2} \lambda_{2}^{2}\right) / 2 \Delta^{2}\right\}\right. \\
\left.+(\sqrt{2} / \pi) A^{-3}\left|A^{2} \lambda_{2}-C \lambda_{1}\right| \exp \left(-\lambda_{1}^{2} / 2 A^{2}\right) \operatorname{erf}\left(\left|A^{2} \lambda_{2}-C \lambda_{1}\right| / \sqrt{2} A \Delta\right)\right] d \theta \\
=\int_{\alpha}^{\beta} I_{1}(\theta) d \theta+\int_{\alpha}^{\beta} I_{2}(\theta) d \theta, \text { say. }
\end{gathered}
$$

We divide the interval $(0,2 \pi)$ into two groups of subintervals. The first group of subintervals includes the $\epsilon$-neighborhood of 0 , and $2 \pi$ and the second lies outside these $\epsilon$-neighborhoods. As in [4], we need further modification of Dunnage's [2] approach which is based on an application of Jensen's theorem [9, page 125] or [8, page 332]. By choosing $\epsilon$ sufficiently small and applying this approach we will be able to show that the expected number of real roots in the $\epsilon$ neighborhood of $0, \pi$ and $2 \pi$ is small and the main contribution to the number of real roots is from those outside these neighborhoods. Indeed $\epsilon$ should be chosen large enough to be able to evaluate the dominant terms of $A^{2}, B^{2}, \ldots$, with the smallest possible error. We chose $\epsilon=n^{-1 / 4}$ and we will show that this choice of $\epsilon$ satisfies both above requirements.

First we let $\theta$ be in either the interval $(\epsilon, \pi-\epsilon)$ or $(\pi+\epsilon, 2 \pi-\epsilon)$. Since the coefficients of $T(\theta)$ are independent normal random variables, we have

$$
A^{2}=\sigma_{1}^{2} \sum_{j=1}^{n_{1}} \cos ^{2} j \theta+\sigma_{2}^{2} \sum_{j=n_{1}+1}^{n} \cos ^{2} j \theta .
$$

Also from [4, page 554] inside this interval we have

$$
\sum_{j=1}^{n_{1}} \cos ^{2} j \theta=\left(n_{1} / 2\right)+O(1 / \epsilon) .
$$

So, combining this with (2.3), we find that

$$
A^{2}=n_{1} \sigma_{1}^{2} / 2+\left(n-n_{1}\right) \sigma_{2}^{2} / 2+O(1 / \epsilon)
$$

Similarly

$$
\begin{aligned}
B^{2} & =\sigma_{1}^{2} \sum_{j=1}^{n_{1}} j^{2} \sin ^{2} j \theta+\sigma_{2}^{2} \sum_{j=n_{1}+1}^{n} j^{2} \sin ^{2} j \theta \\
& =n_{1}^{3} \sigma_{1}^{2} / 6+\sigma_{2}^{2}\left(n^{3} / 6-n_{1}^{3} / 6\right)+O\left(n^{2} / \epsilon\right)
\end{aligned}
$$


and

$$
\begin{gathered}
C=\sigma_{1}^{2} \sum_{j=1}^{n_{1}} j \sin j \theta \cos j \theta+\sigma_{2}^{2} \sum_{j=n_{1}=1}^{n} j \sin j \theta \cos j \theta \\
=O(n / \epsilon) \\
\lambda_{1}=\mu_{1} \sum_{j=1}^{n_{1}} \cos j \theta+\mu_{2} \sum_{j=n_{1}+1}^{n} \cos j \theta-K=0(1 / \epsilon)-K,
\end{gathered}
$$

$$
\lambda_{2}=-\mu_{1} \sum_{j=1}^{n_{1}} j \sin j \theta-\mu_{2} \sum_{j=n_{1}+1}^{n} j \sin j \theta=0(n / c) .
$$

Hence from (2.2)-(2.4) we can obtain

$$
\Delta^{2}=\left\{n_{1} \sigma_{1}^{2} / 2+\left(n-n_{1}\right) \sigma_{2}^{2} / 2\right\}\left\{n_{1}^{3} \sigma_{1}^{2} / 6+\left(n^{3}-n_{1}^{3}\right) \sigma_{2}^{2} / 6\right\}+0\left(n^{3} / \epsilon\right) .
$$

From (2.2) and (2.4)-(2.9) we have

$$
\begin{gathered}
\int_{\epsilon}^{\pi-\epsilon} I_{1}(\theta) d \theta \\
=\left[\frac{n_{1} \sigma_{1}^{2}+\left(n^{3}-n_{1}^{3}\right) \sigma_{2}^{2}}{3\left\{n_{1} \sigma_{1}^{2}+\left(n-n_{1}\right) \sigma_{2}^{2}\right\}}\right] 1 / 2\{1+O(1)\}
\end{gathered}
$$

and

$$
\int_{\epsilon}^{\pi-\epsilon} I_{2}(\theta) d \theta=O\left(n^{1 / 2}\right)
$$

Hence from (2.2), (2.10), (2.11) and since $K=o\left\{K^{2} /\left(n_{1} \sigma_{1}^{2}+n \sigma_{2}^{2}\right)\right\}$ we have

$$
E N(\epsilon, \pi-\epsilon)=E N(\pi+\epsilon, 2 \pi-\epsilon)=\left[\frac{n_{1}^{3} \sigma_{1}^{2}+\left(n^{3}-n_{1}^{3}\right) \sigma_{2}^{2}}{3\left\{n_{1} \sigma_{1}^{2}+\left(n-n_{1}\right) \sigma_{2}^{2}\right\}}\right]^{1 / 2}\{1+o(1)\}
$$

We conclude proof of the theorem by showing that the expected number of real roots outside the intervals in (2.12) is negligible. To this end, let $N(r)$ denote the number of real roots of the random integral equation of the complex variable $z$ of the form

$$
T(z, \omega)-K=0
$$

in the circle $|z|<r$. The upper bound to the number of real roots in the segment of the real axis joining the points $\pm \epsilon$ certainly does not exceed $N(\epsilon)$. Therefore, an upper bound for $N(\epsilon)$ could serve as an upper bound for the number of real roots of $(2.13)$ in the interval $(0, \epsilon)$ and $(2 \pi-\epsilon, 2 \pi)$. The interval $(\pi-\epsilon, \pi+\epsilon)$ can be treated in exactly the same way to give the same result. By Jensen's theorem [9, page 125] or [8, page 332]

$$
N(\epsilon) \log 2 \leq \int_{\epsilon}^{2 \epsilon} r^{-1} N(r) d r
$$




$$
<(2 \pi)^{-1} \int_{0}^{2 \pi} \log \left|\left\{T\left(2 \epsilon e^{i \theta}, \omega\right)-K\right\} /\{T(0)-K\}\right| d \theta .
$$

Now, since $T(0, \omega)=\sum_{j=1}^{n} g_{j}(\omega)$ is normally distributed with mean $\mu=n_{1} \mu_{1}+\left(n-n_{1}\right) \mu_{2}$ and variance $\sigma^{2}=n_{1} \sigma_{1}^{2}+\left(n-n_{1}\right) \sigma_{2}^{2}$, we can see that, for any positive $\nu$,

$$
\begin{aligned}
\operatorname{Prob}\left(-e^{-\nu} \leq T(0)-K<e^{-\nu}\right) & =\left(2 \pi \sigma^{2}\right)^{-\frac{1}{2}} \int_{K-e^{-\nu}}^{K+e^{-\nu}} \exp \left\{-(t-\mu)^{2} / 2 \sigma^{2}\right\} d t \\
& <\left(2 / \pi \sigma^{2}\right)^{\frac{1}{2}} e^{-\nu}
\end{aligned}
$$

In order to find an upper limit for the integrand of (2.14), we notice that

$$
\left|T\left(2 \epsilon e^{i \theta}\right)\right|=\left|\sum_{j=1}^{n_{1}} g_{j} \cos 2 j \epsilon e^{i \theta}\right| \leq n e^{2 n \epsilon} \max \left|g_{j}\right|
$$

where the maximum is taken over $1 \leq j \leq n$. The distribution function of $\left|g_{j}\right|$ is

$$
F(x)=\left\{\begin{array}{cc}
\left(2 / \pi \sigma_{\xi}^{2}\right)^{\frac{1}{2}} \int_{0}^{x} \exp \left\{-\left(t-\mu_{\xi}\right)^{2} / 2 \sigma_{\xi}^{2}\right\} d t & x \geq 0 \\
0 & x<0
\end{array}\right.
$$

where

$$
\xi=\left\{\begin{array}{lll}
1 & \text { for } & 1 \leq j \leq n_{1} \\
2 & \text { for } & n_{1}<j \leq n .
\end{array}\right.
$$

Now since for any positive $\nu$ and $\xi=1,2$

$$
\begin{gathered}
\int_{n e^{\nu}}^{\infty} \exp \left\{-\left(t-\mu_{\xi}\right)^{2} / 2 \sigma_{\xi}^{2}\right\} d t \\
\leq\left(n e^{\nu}-\mu_{\xi}\right)^{-1} \int_{n e^{\nu}}^{\infty}\left(t-\mu_{\xi}\right) \exp \left\{-\left(t-\mu_{\xi}\right)^{2} / 2 \sigma_{\xi}^{2}\right\} d t \\
=\left\{\sigma_{\xi}^{2} /\left(n e^{\nu}-\mu_{\xi}\right)\right\} \exp \left\{-\left(n e^{\nu}-\mu_{\xi}\right) / 2 \sigma_{\xi}^{2}\right\}
\end{gathered}
$$

from (2.17) we have

$$
\begin{gathered}
\operatorname{Prob}\left(\max \left|g_{j}\right|>n e^{\nu}\right) \\
\leq n_{1}\left(2 / \pi \sigma_{1}^{2}\right)^{\frac{1}{2}} \int_{n e^{\nu}}^{\infty} \exp \left\{-\left(t-\mu_{1}\right)^{2} / 2 \sigma_{1}^{2}\right\} d t+\left(n-n_{1}\right)\left(2 / \pi \sigma_{2}^{2}\right)^{\frac{1}{2}} \int_{n e^{\nu}}^{\infty} \exp \left\{-\left(t-\mu_{2}\right)^{2} / 2 \sigma_{2}^{2}\right\} d t \\
<(2 / \pi)^{\frac{1}{2}}\left[\left(n_{1} \sigma_{1} /\left(n e^{\nu}-\mu_{1}\right)\right\} \exp \left\{-\left(n e^{\mu}-\mu_{1}\right) / 2 \sigma_{1}^{2}\right\}\right. \\
\left.+\left\{\left(n-n_{1}\right) \sigma_{2} /\left(n e^{\nu}-\mu_{2}\right)\right\} \exp \left\{-\left(n e^{\nu}-\mu_{2}\right) / 2 \sigma_{2}^{2}\right\}\right]=M, \text { say. }
\end{gathered}
$$


Therefore from (2.16) and (2.18)

$$
\left|T\left(2 \epsilon e^{i \theta}\right)\right|<2 n^{2} \exp (2 n \epsilon+\nu),
$$

except for sample functions in an w-set of measure not exceeding $M$. Hence from (2.15), (2.16) and since for $K=o(\sqrt{n})$

$$
2 n^{2} \exp (2 n \epsilon+\nu)+K<3 n^{2} \exp (2 n \epsilon+\nu)
$$

we obtain

$$
\left|\left\{T\left(2 \epsilon e^{i \theta}, \omega\right)-K\right\} /\{T(0, \omega)-K\}\right|<3 n^{2} \exp (2 n \epsilon-2 \nu)
$$

except for sample functions in an $\omega$-set of measure not exceeding $M+\left(2 / \pi \sigma^{2}\right)^{\frac{1}{2}} e^{-\nu}$. Therefore from (2.14) and (2.19), we find that, outside the exceptional set,

$$
N(\epsilon) \leq \log 3+2 \log n+2 n \epsilon+2 \nu .
$$

Then since $\epsilon=n^{-\frac{1}{4}}$, it follows from (2.20) and for all sufficiently large $n$ that

$$
\operatorname{Prob}\{N(\epsilon)>4 n \epsilon+2 \nu\} \leq M+\left(2 / \pi \sigma^{2}\right)^{\frac{1}{2}} e^{-\nu} .
$$

Let $n^{\prime}=\left[4 n^{\frac{3}{4}}\right]$ be the greatest integer less than or equal to $4 n^{\frac{3}{4}}$, then from $(2.18),(2.21)$ and for all sufficiently large $n$ we obtain

$$
\begin{gathered}
E N(\epsilon)=\sum_{j>0} \operatorname{Prob}\{N(\epsilon) \geq j\} \\
=\sum_{1 \leq j \leq n^{\prime}} \operatorname{Prob}\{N(\epsilon) \geq j\}+\sum_{j \geq 0} \operatorname{Prob}\left\{N(\epsilon)>n^{\prime}+j\right\} \\
\leq n^{\prime}+(2 / \pi)^{\frac{1}{2}}\left[\sigma^{-1} \sum_{j \geq 1} e^{-\frac{j}{2}}+n_{1} \sigma_{1} \sum_{j \geq 1}\left(n e^{\frac{j}{2}}-\mu_{1}\right)^{-1} \exp \left\{-\left(n e^{\frac{j}{2}}-\mu_{1}\right) / 2 \sigma_{1}^{2}\right\}\right. \\
\left.\left.+\left(n-n_{1}\right) \sigma_{2} \sum_{j \geq 1}\left(n e^{\frac{j}{2}}-\mu_{2}\right)^{-1} \exp \left\{-n e^{\frac{j}{2}}-\mu_{2}\right) / 2 \sigma_{2}\right\}\right] \\
=O\left(n^{\frac{3}{4}}\right) .
\end{gathered}
$$

Finally from (2.2), (2.12) and (2.22) we have the proof of the theorem.

\section{ACKNOWLEDGEMENT}

The author would like to thank the referee for his (her) detailed comments which improved the earlier version of this paper. 


\section{REFERENCES}

[1] H. Cramér and M.R. Leadbetter, “Stationary and Related Stochastic Processes", Wiley, New York 1967.

[2] J.E.A. Dunnage, "The number of real zeros of a random trigonometric polynomial", Proc. London Math. Soc. 16, (1966), pp. 53-84.

[3] K. Farahmand, "On the average number of real roots of a random algebraic equation", Ann. Probab. 14, (1986), pp. 702-709.

[4] K. Farahmand, "Level crossings of a random trigonometric polynomial", Proc. Amer. Math. Soc. 111, (1991), pp. 551-557.

[5] K. Farahmand, "Real zeros of random algebraic polynomial", Proc. Amer. Math. Soc. 113, (1991), pp. 1077-1084.

[6] I.A. Ibragimov and N.B. Maslova, "Average number of real roots of random polynomials", Soviet Math. Dokl. 12, (1971), pp. 1004-1008.

[7] S.O. Rice, "Mathematical theory of random noise", Bell System Tech. J. 25, (1945), pp. 46-156.

[8] W. Rudin, "Real and Complex Analysis", 2nd edition, McGraw-Hill 1974.

[9] E.C. Titchmarsh, "The Theory of Functions", 2nd edition, Oxford University Press 1939.

[10] J.E. Wilkins, Jr., “Mean number of real zeros of a random trigonometric polynomial”, Proc. Amer. Math. Soc. 111, (1991), pp. 851-863.

[11] J.E. Wilkins, Jr., "An asymptotic expansion for the expected number of real zeros of a random polynomial", Proc. Amer. Math. Soc. 103, (1988), pp. 1249-1258. 


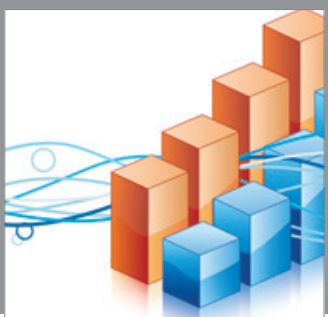

Advances in

Operations Research



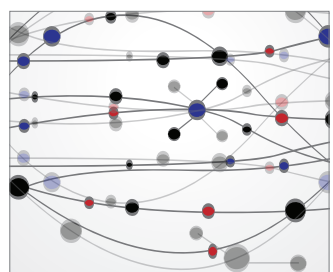

\section{The Scientific} World Journal
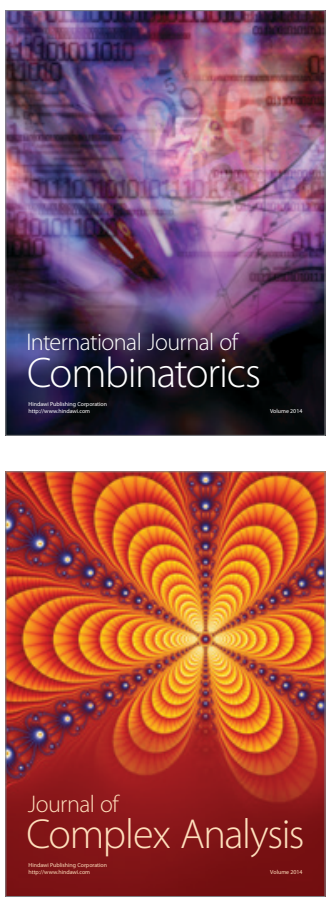

International Journal of

Mathematics and

Mathematical

Sciences
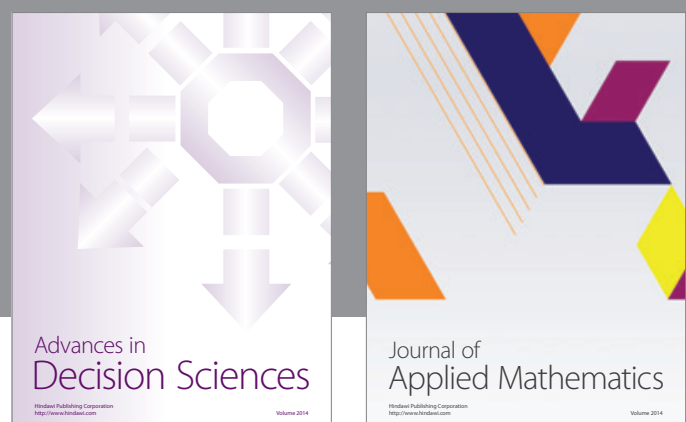

Journal of

Applied Mathematics
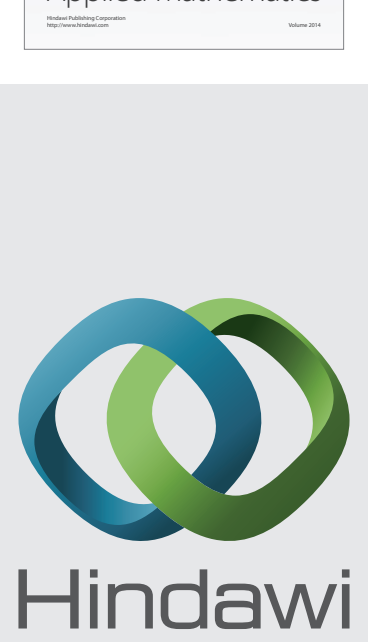

Submit your manuscripts at http://www.hindawi.com


Mathematical Problems in Engineering
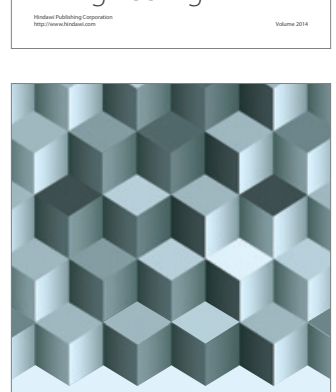

Journal of

Function Spaces
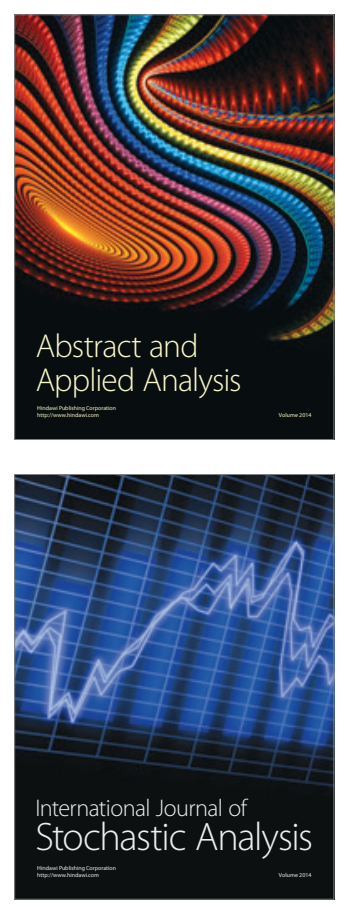

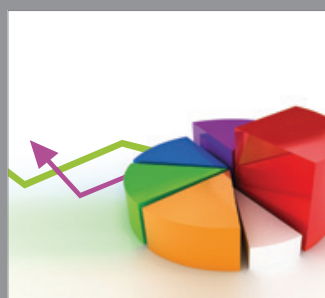

ournal of

Probability and Statistics

Promensencen
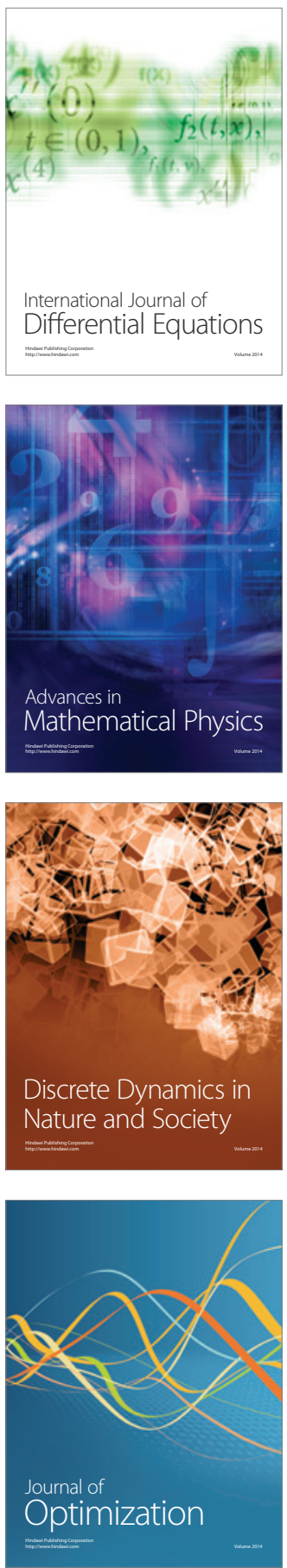